\title{
ON THE REPRESENTATION OF AN INTEGER AS THE SUM OF THREE FOURTH POWERS
}

\section{PARTHASARATHY}

1. Introduction. Let $r_{s, k}(N)$ denote the number of representations of a positive integer $N$ as the sum of $s k$ th powers of positive integers. S. Chowla and S. S. Pillai [2] proved that, for infinitely many $N$,

$$
r_{k, k}(N)>c_{1} \log \log N
$$

where $c_{1}$ (as well as $c_{2}, c_{3}, \cdots$ in what follows) is a constant independent of $N$. P. Erdös [5] improved this and proved in an elementary way that, for infinitely many $N$,

$$
r_{k, k}(N)>\exp \left(c_{2} \frac{\log N}{\log \log N}\right) .
$$

However, for $s<k$ only particular cases have been studied. The only results I have come across are the following two due respectively to S. S. Pillai and S. Chowla (see Chowla $[1$, p. 122]). For an infinity of $N, n$,

$$
\boldsymbol{r}_{2,3}(N)>c_{3} \log \log N
$$

and

$$
r_{3,4}(n)>c_{4} \frac{\log n}{\log \log n} .
$$

In this note (\$3) I prove that, for infinitely many $N$,

$$
r_{3,4}(N)>\exp \left(c_{5} \frac{\log N}{\log \log N}\right) .
$$

The proof is elementary and entirely different from that of Chowla.

2. Lemmas. For the proof of our theorem we require some lemmas. In what follows, all the letters denote positive integers.

LeMma 1. Let $p_{1}, p_{2}, \cdots, p_{r}$ be the $r$ consecutive primes $>78$ and belonging to the arithmetic progression $6 n+1$. Then, corresponding to every $p_{i}$, there is a constant $k_{i}<22464$ such that $n_{i}^{2}=\left(p_{i} k_{i}\right)^{2}$ has a representation of the form $x_{i}^{2}+3 y_{i}^{2}$ satisfying the conditions that $x_{i}>7 y_{i}>0$ and $p_{i}$ does not divide $x_{i} y_{i}$.

Received by the editors March 10, 1952. 
Proof. It is well known (Euler [4]) that every prime of the form $6 n+1$ is of the form $x^{2}+3 y^{2}$. Let $p_{i}=\xi_{i 1}^{2}+3 \eta_{i 1}^{2}$. The inequalities which follow are easily seen to be strict inequalities.

In case $\xi_{i 1}>2 \eta_{i 1}$ we write $q_{i}=p_{i}$; while in case $\xi_{i 1}<2 \eta_{i 1}$ we write $q_{i}=p_{i}\left(\lambda^{2}+3\right)$ where $\lambda$ is the integer such that

$$
\frac{\xi_{i 1}}{\eta_{i 1}}<\lambda<\frac{\xi_{i 1}}{\eta_{i 1}}+1<3 .
$$

The number $q_{i}$ defined in the second case has the representation

$$
q_{i}=\xi_{i 2}^{2}+3 \eta_{i 2}^{2} \text { (say) }
$$

where

$$
\xi_{i 2}=\lambda \xi_{i 1}+3 \eta_{i 1} \text { and } \eta_{i 2}=\lambda \eta_{i 1}-\xi_{i 1} .
$$

From (2.1) and (2.2) we get

$$
\xi_{i 2}>2 \eta_{i 2} \text {. }
$$

We see from (2.3) that the representation

$$
q_{i}^{2}=\xi_{i 3}^{2}+3 \eta_{i 3}^{2}, \quad 0<\xi_{i 3}<\eta_{i 3},
$$

is possible, $q_{i}$ being defined by either $q_{i}=p_{i}$ or $q_{i}=p_{i}\left(\lambda^{2}+3\right)$. Hence

$$
16 q_{i}^{2}=\left(6 \eta_{i 3}-2 \xi_{i 3}\right)^{2}+3\left(2 \xi_{i 3}+2 \eta_{i 3}\right)^{2}=\xi_{i 4}^{2}+3 \eta_{i 4}^{2} \text { (say). }
$$

From (2.4) and (2.5) we get

$$
0<\eta_{\text {i4 }}<\xi_{\text {in }}<3 \eta_{\text {is. }}
$$

Now

$$
144\left(\xi_{i 4}^{2}+3 \eta_{i 4}^{2}\right)=\left(6 \xi_{i 4}+18 \eta_{i 4}\right)^{2}+3\left(6 \xi_{i 4}-6 \eta_{i 4}\right)^{2}=\xi_{i b}^{2}+3 \eta_{i b}^{2} \text { (say). }
$$

We verify that

$$
\xi_{i 6}>3 \eta_{i 5}
$$

We now consider two cases.

(i) $\xi_{i b}>7 \eta_{i 5 .}$. In this case we write $n_{i}=48 q_{i}$.

(ii) $7 \eta_{i b}>\xi_{i b}>3 \eta_{i b}$. Now

$$
2704\left(\xi_{i 5}^{2}+3 \eta_{i b}^{2}\right)=\left(46 \xi_{i 5}+42 \eta_{i b}\right)^{2}+3\left(46 \eta_{i b}-14 \xi_{i 5}\right)^{2} .
$$

We can verify that

$$
46 \xi_{i b}+42 \eta_{i b}>7\left|46 \eta_{i b}-14 \xi_{i b}\right| .
$$


In case (ii) we write $n_{i}=2496 q_{i}$. The number $n_{i}$ defined as above for the two cases is such that $n_{i}^{2}$ has a representation $x_{i}^{2}+3 y_{i}^{2}, x_{i}>7 y_{i}>0$. Using the fact that $p_{i}>78$ and does not divide $\xi_{i 1} \eta_{i 1}$ we can show that $p_{i}$ does not divide $x_{i} y_{i}$.

Lemma 2. Let $n_{i}, i=1,2, \cdots, r$, be as in Lemma 1. Then the number $n_{j_{1}}^{2} n_{j_{2}}^{2} \cdots n_{j_{k}}^{2}, 0<j_{1}<j_{2}<\cdots<j_{k} \leqq r, 1 \leqq k<r$, has a representation of the form $x^{2}+3 y^{2}$ with $x>7 y>0$ and such that $p_{j_{\mathrm{e}}}, s=1,2, \cdots, k$, does not divide $x y$.

The proof is by induction on $k$.

LEMma 3. Let $R_{1}(N)$ denote the number of representations of an integer $N$ in the form $x^{2}+3 y^{2}, x>7 y>0$. Then, for infinitely many $N$,

Proof. Let

$$
R_{1}(N)>\exp \left(c_{6} \frac{\log N}{\log \log N}\right) .
$$

$$
\begin{aligned}
& A=n_{1} n_{2} \cdots n_{r} \\
& B=n_{j_{1}} n_{j_{2}} \cdots n_{j_{k}}, \quad 0<j_{1}<j_{2}<\cdots<j_{k} \leqq r, 1 \leqq k<r \text {, } \\
& A=B C \text { and } N=A^{2} \text {, }
\end{aligned}
$$

where the $n$ 's are as in Lemma 1.

By Lemma 2, $B$ has a representation $x^{2}+3 y^{2}, x>7 y>0$, such that $p_{j_{z}}, s=1,2, \cdots k$, does not divide $x y$. This gives a representation $(C x)^{2}+3(C y)^{2}$ for $N$ satisfying the conditions of Lemma 3. No two $B$ 's can give rise to the same representation. For, if $B$ and $B^{\prime}$ give the same representation we shall have an equality of the type $C x=C^{\prime} x^{\prime}$. If $C \neq C^{\prime}$ there is a prime $p$ which divides (say) $C$ but not $C^{\prime}$. So $p$ divides $x^{\prime}$. Also $p$ divides $B^{\prime}$ since it does not divide $C^{\prime}$. This is a contradiction since the representation of $B^{\prime}$ which we consider is such that if $p$ divides $B^{\prime}$ then $p$ does not divide $x^{\prime}$.

Hence

$$
R_{1}(N) \geqq \sum_{B} 1=2^{r}-2 .
$$

Now, by the prime number theorem for arithmetic progressions, $p_{k}<c_{7} k \log k$ and

so that

$$
\log N=2\left(\log n_{1}+\cdots+\log n_{r}\right)<c_{8} r \log r
$$

$$
r>c_{9} \frac{\log N}{\log \log N} \text {. }
$$


From (2.6) and (2.7) the conclusion of the lemma follows.

LEMMA 4. Let $R_{2}(N)$ denote the number of representations of an integer $N$ in the form $\xi^{2}-\xi \eta+\eta^{2}, \xi>4 \eta>0$. Then

$$
R_{2}(N)>\exp \left(c_{6} \frac{\log N}{\log \log N}\right)
$$

for infinitely many $N$.

Proof. Writing $\xi=x+y$ and $\eta=2 y$ and taking $N$ as in Lemma 3, we get

$$
R_{2}(N)=R_{1}(N)>\exp \left(c_{B} \frac{\log N}{\log \log N}\right) .
$$

3. Theorem. For infinitely many $M$,

$$
r_{3,4}(M)>\exp \left(c_{5} \frac{\log M}{\log \log M}\right) .
$$

Proof. Consider the identity [3]

$$
\left(k^{2}-2 k\right)^{4}+(2 k-1)^{4}+\left(k^{2}-1\right)^{4}=2\left(k^{2}-k+1\right)^{4} .
$$

Writing $k=\xi / \eta$ and multiplying by $\eta^{8}$ this becomes

$$
\left(\xi^{2}-2 \xi \eta\right)^{4}+\left(2 \xi \eta-\eta^{2}\right)^{4}+\left(\xi^{2}-\eta^{2}\right)=2\left(\xi^{2}-\xi \eta+\eta^{2}\right)^{4} .
$$

If $N$ is as in Lemmas 3 and 4 , there are $R_{2}(N)$ pairs of values of $\xi$ and $\eta, \xi>4 \eta>0$, for which the right-hand side of (3.1) can be fixed equal to $M=2 N^{4}$. Thus we get $R_{2}(N)$ representations of $N$ in the the form $x_{1}^{4}+x_{2}^{4}+x_{3}^{4}$.

Now if $\xi>4 \eta$ the greatest and the smallest of the expressions on the left-hand side of (3.1) are respectively $\xi^{2}-\eta^{2}$ and $2 \xi \eta-\eta^{2}$. If $\xi_{1}, \eta_{1}$ and $\xi_{2}, \eta_{2}$ give the same representation, then

$$
\begin{aligned}
\xi_{1}^{2}-\xi_{1} \eta_{1}+\eta_{1}^{2} & =\xi_{2}^{2}-\xi_{2} \eta_{2}+\eta_{2}^{2}, \\
\xi_{1}^{2}-\eta_{1}^{2} & =\xi_{2}^{2}-\eta_{2}^{2},
\end{aligned}
$$

and

$$
2 \xi_{1} \eta_{1}-\eta_{1}^{2}=2 \xi_{2} \eta_{2}-\eta_{2}^{2}
$$

These relations are easily seen to be inconsistent unless $\xi_{1}=\xi_{2}$ and $\eta_{1}=\eta_{2}$. Hence all the representations given by different pairs of values of $\xi$ and $\eta$ are distinct. 
It follows that

$$
\begin{aligned}
r_{3,1}(M) & \geqq R_{2}(N) \\
& >\exp \left(c_{6} \frac{\log N}{\log \log N}\right) \\
& >\exp \left(c_{6} \frac{\log M}{\log \log M}\right) . \\
& \text { REFERENCES }
\end{aligned}
$$

1. S. Chowla, Contributions to the analytic theory of numbers II, J. Indian Math. Soc. vol. 20 (1933) pp. 120-128.

2. S. Chowla and S. S. Pillai, Hypothesis-K of Hardy and Littlewood, Math. Zeit. vol. 41 (1936) pp. 537-540.

3. L. E. Dickson, History of the theory of numbers, vol. 2, p. 656.

4. - History of the theory of numbers, vol. 3, p. 4.

5. P. Erdös, On the representation of an integer as the sum of $k$ k-th powers, J. London Math. Soc. vol. 11 (1936) pp. 133-136.

Rayanujan Instrtute of Mathematics 